\title{
Epitaxially grown sexiphenyl nanocrystals on the organic $\mathrm{KAP}(010)$ surface
}

Thomas Haber ${ }^{1}$, Roland Resel ${ }^{1, *}$, Annette Thierry ${ }^{2}$, Marcello Campione ${ }^{3}$, Adele Sassella ${ }^{3}$ and Massimo Moret $^{3}$

${ }^{1}$ Institute of Solid State Physics, Graz University of Technology, Petersgasse 16, A-8010 Graz, Austria

${ }^{2}$ Institut Charles Sadron, Centre National de la Recherche Scientifique 23 rue du Loess, F-67034 Strasbourg, France

${ }^{3}$ Dipartimento di Scienza dei Materiali and CNISM, Università di Milano Bicocca, via Cozzi 53, I-20125 Milano, Italy

* corresponding author.

phone: +43 316873 8476, fax: +43 3168738466 ,

e-mail address: roland.resel@tugraz.at (R. Resel) 


\begin{abstract}
:
Nanocrystals of the organic molecule sexiphenyl are grown on the (010) cleavage plane of potassium hydrogen phthalate (KAP). The single crystalline organic surface is composed exclusively by phenyl rings and displays two distinct directions of aromatic rows forming surface corrugations. Sexiphenyl crystals grow epitaxially ordered with the (20-3) plane parallel to $\mathrm{KAP}(010)$ with the long molecular axes of the molecule aligned along one specific surface corrugation; empirical force field calculations confirm the experimentally observed epitaxial alignment of the sexiphenyl crystals. The sexiphenyl crystals grow as elongated islands, which can be shown to be of single crystalline nature.
\end{abstract}

PACS: 68.55.am, 68.37.Lp, 68.35.bm, 61.66.Hq

Keywords: sexiphenyl, organic heteroepitaxy, organic thin film morphology 


\section{Introduction}

Fundamental research on mechanisms of aggregation of organic molecular materials is an important step for the understanding of film growth mechanisms and, in succession, for controlling the growth [13]. The effort in this field is basically motivated by the potential of organic semiconductors in diverse electronic devices (light-emitting devices, photovoltaic cells, and field-effect transistors). The strict dependence of device performances on molecular orientation is due to the anisotropy of the molecules, the molecular crystals, and the correlated electronic and optical properties [4-6]. Therefore the ability to control molecular orientation and crystallographic order of the molecular materials is highly beneficial for improving device performances. Moreover, in a device the crystal properties such as crystal structure, grain size and strain show distinct influence. In particular sexiphenyl, which is a rod like molecule and a blue light emitting organic semiconductor with an outstanding high quantum yield, shows blue light emission perpendicular to the long molecular axis (LMA) [7,8]. Moreover, the sexiphenyl molecular crystal, a layered crystal with the so-called herringbone arrangement of molecules within each single layer (herringbone layer), is reported to be a wave guiding crystal in the plane of the herringbone layers [9]. The waveguide effect abruptly ends at grain boundaries [10]. Therefore ordered, single crystalline structures are of particular interest for future applications.

Recently, the field of interest of organic molecular films has expanded beyond the growth on metallic, semi-conducting and insulating surfaces to organic heteroepitaxy, often realized by a multilayer growth [11-14]. In the present study we follow this way, using the highly ordered cleavage plane of potassium hydrogen phthalate - KAP(010) - as organic template for the growth study. This surface is molecularly flat and shows phenyl rings pointing out of the crystal [15]. Furthermore, it displays two distinct directions of surface corrugations running along $<101>$ and [001]. The surface characteristics, the oriented phenyl rings as well as the surface corrugations, are likely to induce an epitaxial growth of sexiphenyl - what was finally observed.

\section{Experimental}

The KAP single crystals (purchased from Ekspla, Vilnius, Lithuania) were mechanically cleaved in air immediately before introduction into the growth chamber for organic molecular beam epitaxy [16]. High purity sexiphenyl (p6P, $\mathrm{C}_{36} \mathrm{H}_{26}$ ) purchased from Tokyo Chemical Industries was deposited at a base pressure of $10^{-7}$ Torr, a growth rate of $0.3 \mathrm{~nm} \mathrm{~min}^{-1}$, and a substrate temperature of $300 \mathrm{~K}$. Films with nominal thicknesses between 10 to $20 \mathrm{~nm}$ were prepared, as monitored by a quartz microbalance $[17]$. 
The surface morphology of the films was investigated by atomic force microscopy (AFM) using a Digital Instrument Nanoscope IIIa MMAFM equipped with E- and J-type scanners. The out-of-plane orientation as well as the in-plane alignment of the sexiphenyl crystallites were determined by $\mathrm{x}$-ray diffraction (XRD) techniques using a PHILIPS X'PERT system equipped with an ATC3 cradle operated with $\mathrm{CrK} \alpha$ radiation and a secondary monochromator. The measurements were based on the orthorhombic crystal structure of KAP with lattice constants of $\mathrm{a}=9.61 \AA, \mathrm{b}=13.33 \AA, \mathrm{c}=6.48 \AA[18]$, and on the monoclinic structure of sexiphenyl with $\mathrm{a}=8.09 \AA, \mathrm{b}=5.57 \AA, \mathrm{c}=26.24 \AA, \beta=98.17 \AA$ [19]. The software packages POWDER CELL, MERCURY and STEREOPOLE were used for data analysis [20,21]

Atom-atom potential calculations were performed to study the correlation between orientation of p6P crystals on the KAP(010) plane in terms of potential energy. A modified version of the AutoDock3 molecular docking package [22] was used in combination with the UNI empirical force field without point charges on atoms [23]. A simulation box with $351^{3}$ grid points and a grid spacing of $0.217 \AA$ was used in order to sample the interaction potential with a fine grid. Preliminary simulations with $251^{3}$ grid points were satisfactory but definitely less accurate on determining the potential energy minima. The $\mathrm{KAP}(010)$ substrate surface was modeled with a slab composed by $11 \times 2 \times 17$ unit cells for $a, b$ and $c$ axis, respectively, giving rise to a total of 1728 formula units of $\mathrm{C}_{8} \mathrm{H}_{5} \mathrm{O}_{4} \mathrm{~K}$ and 31104 atoms. The sexiphenyl crystal was modeled by two herringbone layers of sexiphenyl molecules representing a slice of the (20-3) plane. Two different sets of simulations runs were performed; the first one was based on a p6P slab consisting of three molecular layers parallel to (20-3) with $3 / 4 / 3$ and $4 / 3 / 4$ molecules for the two herringbone layers (i.e. 7 molecules contact the plane of the substrate) (996 docking runs), the second one comprised a stacking of 5/4/3 and 4/3/4 molecules (i.e. 9 molecules contact the plane of the substrate, 1092 docking runs) to check the relevance of the lateral size of the crystallite. For the latter only the 982 docked structures with the 5+4 contact p6P molecules were used for final statistics.

For transmission electron microscopy (TEM) the films were covered with a uniform carbon film and detached from the KAP substrate. Bright field images, diffraction patterns and high resolution images were recorded by a PHILIPS CM12 electron microscope operating at energy of $120 \mathrm{keV}$.

\section{Results and discussion}

An AFM image is shown in Figure 1 where small elongated islands of sexiphenyl are observed. The islands have a tabular shape with an average height of $40 \mathrm{~nm}$, a width of $80 \mathrm{~nm}$ and a characteristic length of $300 \mathrm{~nm}$. The surface is not completely covered by these islands, also some dark areas less than $100 \mathrm{~nm}$ appear. The elongated islands are aligned along two preferred azimuthal directions which are inclined by $70^{\circ}\left( \pm 10^{\circ}\right)$ relative to each other. 
The crystallographic properties of the islands were determined by XRD specular scans, the results are shown in Figure 2a. The presented peak is identified as the 20-3 diffraction of p6P and indicates that the crystals grow with the (20-3) plane parallel to the surface. There are several examples of epitaxially grown p6P films where this (20-3) plane is found parallel to the substrate surface; generally this plane of p6P is formed parallel to the substrate surface when either the interaction of the p6P molecules with the substrate is rather weak like for $\mathrm{KCl}(100)$ or surface corrugations induce the formation of this specific plane of p6P [24,25]. The film mosaicity is probed by a rocking curve of this diffraction peak (see Figure 2b). As a reference, the rocking curve of the $\mathrm{KAP}(030)$ peak is shown in the same graph as it reflects the experimental broadening. The FWHM of the p6P peak is $1.3^{\circ}$ while in case of the KAP a peak width of $0.3^{\circ}$ is observed. Therefore, a mosaicity of approximately $1^{\circ}$ can be concluded, which is a good value for organic-organic heteroepitaxy [26].

\section{Figure 2}

The epitaxial alignment of the p6P crystals was determined by recording a set of pole figures. One pole figure is representatively shown in Figure 3; it is measured at $2 \Theta=42.40^{\circ}$ to detect the $\{21-3\}$ planes of p6P crystals. Enhanced pole densities (EPDs) of both the substrate and the film are identified. Due to the fact that the 140 and 012 peaks of the KAP substrate are at close $2 \Theta$ angles $\left(42.64^{\circ}\right.$ and $42.66^{\circ}$, respectively), EPD of these diffraction peaks appear in the pole figure, too. Four diffraction features of the sexiphenyl crystals arise which can be assigned to 21-3 and 2-1-3 originated from the (20-3) orientation of the crystals. Two distinct alignments of the sexiphenyl crystals are present, the belonging diffraction features of one crystal alignment is marked by the grey arrows. The two crystal alignments are twisted by an angle of $65^{\circ} \pm 5^{\circ}$ relative to each other, which agrees quite well with the inclination angle of the elongated islands observed by AFM. The in-plane mosaicity can be estimated from the $\varphi$ broadening of the (21-3) reflections and amounts to $15^{\circ}$, a considerable larger value than the in-plane mosaicity of the KAP substrate which determined smaller than $1^{\circ}$.

Based on the $(\varphi / \psi)$ positions of the EPDs, the epitaxial relationships could be determined as: $(010)_{\mathrm{KAP}}$ $\left\|(20-3)_{\mathrm{p} 6 \mathrm{P}} \&[ \pm 101]_{\mathrm{KAP}}\right\|[203]_{\mathrm{p} 6 \mathrm{P}}$. Please note that the two crystal directions $[ \pm 101]_{\mathrm{KAP}}$ mirror two equivalent $\mathrm{p} 6 \mathrm{P}$ orientations in-plane-rotated by $68^{\circ}$ to each other, which is in excellent agreement with the angle between the two azimutal directions of the elongated islands observed in the AFM images. 
The epitaxial matrix [27] can be determined: $\mathrm{C}_{11}=4.71, \mathrm{C}_{12}=4.71, \mathrm{C}_{21}=-0.32$ and $\mathrm{C}_{22}=0.71 \mathrm{using}$ the rectangular surface unit cell of KAP with $\mathrm{a}_{1}=9.61 \AA, \mathrm{a}_{2}=6.48 \AA$ and of p6P with $\mathrm{b}_{1}=54.6 \AA$ and $\mathrm{b}_{2}=5.57 \AA$. The lattices are hence incommensurable with each other [28], but epitaxy occur due to surface corrugations of the KAP substrate as it is observed also for other molecules [11,29]. This example shows that besides the well known 2-dimensional lattice matching also other mechanisms exist which can induce organic epitay. The effect of surface corrugations to the epitaxial order of rod-like molecules is also observed on inorganic surfaces which show comparable strong corrugation like oxygen reconstructed $\mathrm{TiO}_{2}(110)$ and $\mathrm{Cu}(110)(2 \times 1) \mathrm{O}[25,30]$, but also systems with weak surface corrugation depth [e.g. $\mathrm{KCl}(110)]$ show a clear effect on the epitaxial order [24].

\section{Figure 3}

Based on the crystallographic information a tentative scheme of the interface of 6P film and KAP at a molecular level can be drawn, as shown in Figure 4. The p6P molecules are aligned along the [101 $]_{\text {KAP }}$ direction which is the direction of grooves caused by the emerging phenyl rings. The lateral spacing between the grooves is $5.38 \AA$. (Fig 4 - center and bottom). Considering the 20-3 plane of sexiphenyl the molecular spacing between phenyl rings of neighbouring molecules is $5.57 \AA$, which results in a misfit of only $4 \%$ in the direction of a herringbone layer. The more pronounced surface corrugation (Fig 4 - top) running along [001] KAP does not drive the molecular orientation of the p6P crystals. However, the surface corrugation is not the only property driving the observed molecular arrangement; indeed, the aromatic planes of the surface phenyl rings pointing out of the surface can contribute. Directed interactions between phenyl rings can induce specific alignments of the p6P molecules.

Figure 4

To clarify the role of thermodynamics on the formation of epitaxial order, potential energy calculations were performed. The (20-3) plane of p6P was randomly rotated and translated, then left to interact with the KAP(010) surface. The results are summarized and depicted in Figure 5. As expected, the potential energy resulted to be strongly dependent on the azimuthal angle of the p6P crystal (here defined as the angle between the long molecular axis of p6P) with [100 $]_{\text {KAP }}$ direction (Fig.4). After taking into account the symmetry of the system the only and sharp energy minimum obtained is for azimutal orientations of about $34^{\circ}$ (Fig. 5a) which represents exactly the $[ \pm 101]_{\mathrm{KAP}}$ directions of the molecular grooves. 
Moreover, a necessary and simultaneous condition required for reaching a good (negative) interaction energy is the tilt angle $(\eta)$ between the reciprocal space $[20-3]_{\mathrm{p} 6 \mathrm{P}}^{*}$ and $[010]^{*}{ }_{\mathrm{KAP}}$ directions, i.e the normals to the crystallographic planes. As can be seen in Figure 5b, only at very small $\eta$ tilt angles a good potential energy can be obtained. These results of the calculations confirms the experimental observations: an alignment of the LMA of p6P with small deviations from $[ \pm 101] \mathrm{KAP}$ as observed by the pole figure measurements and small $\eta$ tilt angles as observed by the rocking curves.

The other feature of Figure 5a worth mentioning is the secondary energy minimum found at azimutal angles close to $90^{\circ}$, hence corresponding to the LMA of p6P parallel to [001 $]_{\mathrm{KAP}}$. As stated previously, this direction exhibits the widest grooves but experimentally there were no crystallites aligned parallel to this direction. The total absence of crystallites with these specific alignment can be understood in terms of Maxwell-Boltzmann statistics which gives a negligible probability of occurence for this epitaxial orientation for a cluster of few molecules.

It is remarkable that this energy calculation, based on rigid models, predicts correctly the epitaxial orientation of $(20-3)_{\mathrm{p} 6 \mathrm{P}}$ plane on $(010)_{\mathrm{KAP}}$. Only weak distortions from their bulk crystallographic structure can be expected for both KAP substrate and p6P. It has to be pointed out that the lattices are incommensurable and that a lattice mismatch calculation based on the two contact planes $(010)_{\mathrm{KAP}}$ and $(20-3)_{\mathrm{p} 6 \mathrm{P}}$ reveals only weak minima close to the observed alignment. Thererefore, even though the present simulation is much more computationally demanding than lattice geometry calculations [31] the results seems to be more reliable and robust, since they probably take into account the most relevant driving force, i.e. local interactions given by the crystallo-chemical properties; in other words, more subtle structural details have to be explored and analyzed in order to interpret or even predict organicorganic heteroepitaxy.

Figure 5

The real structure of the sexiphenyl crystals, especially the correlation between crystal structure and island morphology are obtained by TEM techniques.

Figure 6

Figure 6a shows a defocused image of a 10nm thick film. As in the case of AFM investigations the morphology of elongated islands with the two distinct alignments of the islands are clearly observed. Although the width of the p6P islands appears in this TEM pattern much thinner than in the AFM 
micrograph, its lateral dimensions are in agreement, since in case of AFM the spatial extention of the tips causes always a lateral broadening of island structures. Diffraction diagrams over large areas, not presented here, confirm the (20-3) orientation of the crystals already identified by XRD. Besides the elongated island structure also islands with plate like morphology are found. To clarify the nature of these plates, selected area diffraction pattern was taken from one of these islands. The investigated area is marked with a grey spot in Fig.6a and the corresponding diffraction pattern is shown in Fig.6b. Indexation of the diffraction pattern reveals that this island represents also a p6P crystallite, however with (001) as contact plane i.e. a crystal orientation with up-right standing molecules. Although this orientation was too weak to be identified by XRD, it explains the dark areas observed in the AFM image (Fig. 1). Diffraction patterns over large areas reveal that on average the (001)-oriented crystallites are aligned isotropically (2D-powder). A high resolution image of elongated islands is shown in Figure 6c, which shows a layered appearance as internal structure; layers are parallel to the needle direction. These layers correspond to the herringbone monolayers with a thickness of $2.6 \mathrm{~nm}$, reflecting the single crystalline nature of the needles. Single crystals with such large lateral extension are also observed for p6P on $\mathrm{KCl}(100)$ and for quaterthiophene on $\operatorname{KAP}(010)[32,33]$.

\section{Conclusions}

The surface of $\operatorname{KAP}(010)$ serves as a good organic template for the controlled growth of crystalline sexiphenyl. The molecules condense in the typical bulk phase with mainly the (20-3) as contact plane, forming tabular crystals with an elongated island like morphology. Only a small fraction of plate-like islands made of standing molecules are formed. The elongated islands are shown to be single crystals with typical dimensions of about 40nm height, $10 \mathrm{~nm}$ width and several $100 \mathrm{~nm}$ of length. The epitaxial order can be described by the long molecular axes of sexiphenyl aligned along the $<101>$ grooves of the surface. The lateral distances between the adapted surface corrugation of KAP(010) and sexiphenyl(203) have a mismatch of only 4\%. Potential energy calculations based on the experimentally observed (203) orientation correctly predicts the adapted observed in-plane alignment of the sexiphenyl crystals. This fact reveals that the interface between the sexiphenyl crystals and the KAP suface is formed by the two involved crystallographic planes, therefore the (20-3) plane can be assigned as contact plane of the sexiphenyl molecules to the $\mathrm{KAP}(010)$ lattice.

\section{Acknowledgment}

The authors thank Patrick Schultz, IGBMC, Illkirch, France for high resolution TEM investigations. The financial support of the Italian-Austrian Exchange project (project no. A-15/2004) and of the 
Austrian Science Foundation (FWF) within the National Research Network "Interface Controlled and Functionalised Organic Films" is acknowledged.

\section{References}

[1] J. Fraxedas, Adv. Mater. 14 (2002) 1603.

[2] G. Witte, C. Wöll, J. Mater. Res. 19 (2004) 1889.

[3] F. Schreiber, phys. Stat. Sol. (a) 201 (2004) 1037.

[4] N. Karl, Synthetic Metals 133-134 (2003) 649.

[5] N. Koch, Chem. Phys. Chem. 8 (2007) 1438.

[6] F. Balzer, H.-G. Rubahn, Adv. Funct. Mater. 15 (2005) 17.

[7] J. Stampfl, S. Tasch, G. Leising, U. Scherf, Synth. Met. 71 (1995) 2125.

[8] E. Zojer, N. Koch, P. Puschnig, F. Meghdadi, A. Niko, R. Resel, C. Ambrosch-Draxl, M. Knupfer, J. Fink, J. L. Bredas, G. Leising, Phys. Rev. B 61 (2000) 16538.

[9] F. Balzer, V. G. Bordo, A. C. Simonsen, H. -G. Rubahn, Appl. Phys. Lett. 82 (2003) 10.

[10] H. Yanagi, T. Morikawa, Appl. Phys. Lett 75 (1999) 187.

[11] M. Campione, A. Sassella, M. Moret, A. Papagni, S. Trabattoni, R. Resel, O. Lengyel, V. Marcon, and G. Raos, J. Am. Chem. Soc. 128 (2006) 13378.

[12] A. Sassella, A. Borghesi, M. Campione, S. Tavazzi, C. Goletti, G. Bussetti, P. Chiaradia, Appl. Phys. Lett. 89 (2006) 261905.

[13] G. Koller, S. Berkebile, J. R. Krenn, F. P. Netzer, M. Oehzelt, T. Haber, R. Resel, M. G. Ramsey, Nanoletters 6 (2006) 1207.

[14] M. Oehzelt, G. Koller, J. Ivanco, S. Berkebile, T. Haber, R. Resel, F.P. Netzer, M.G. Ramsey Adv. Mater. 18 (2006) 2466.

[15] J. Borc, K. Sangwal, Surf. Sci. 555 (2004) 1.

[16] R. Tubino, A. Borghesi, L. Dalla Bella, S. Destri, W. Porzio, A. Sassella, Opt. Materials 9, (1998) 437.

[17] M. Campione, M. Cartotti, E. Pinotti, A. Sassella, and A. Borghesi, J. Vac. Sci. Techn. A 22 (2004) 482.

[18] T. A. Eremina, N. G. Furmanova, L. F. Malakhova, T. M. Okhrimenko, V. A. Kuznetsov, Crystallogr. Rep. 38 (1993) 554.

[19] K. N. Baker, A. V. Fratini, T. Resch, H. C. Knachel, W. W. Adams, E. P. Socci, B. L. Farmer, Polymer 34, 1571 (1993).

[20] W. Kraus, G. Nolze, J. Appl. Crystallogr. 29 (1996) 301.

[21] I. Salzmann, R. Resel, J. Appl. Crystallogr. 37 (2004) 1029.

[22] G.M. Morris, D.S. Goodsell,, R.S. Halliday, R. Huey, W.E. Hart, R.K. Belew, A.J. Olson, J. Comp. Chem. 19 (1998) 1639.

[23] A. Gavezzotti, Molecular aggregation: structure analysis and molecular simulation of crystals and liquids, Oxford University Press, USA (2007) 215.

[24] T. Haber, A. Andreev, A. Thierry, H. Sitter, M. Oehzelt, R. Resel, J. Cryst. Growth 284 (2005) 209.

[25] G. Koller, S. Berkebile, J. Krenn, G. Tzvetkov, G. Hlawacek, O. Lengyel, F. P. Netzer, C. Teichert, R. Resel, M. G. Ramsey, Adv. Mater. 16 (2004) 2159.

[26] G. Koller, S. Berkebile, J. R. Krenn, F. P. Netzer, M. Oehzelt, T. Haber, R. Resel, M. G. Ramsey Nanoletters 6 (2006) 1207. 
[27] S. C. B. Mannsfeld, T. Fritz, Phys. Rev. B 71 (2005) 1.

[28] D. E. Hooks, T. Fritz, M. D. Ward, Adv. Mater. 13 (2001) 227.

[29] V. Marcon, G. Raos, M. Campione, A. Sassella, Crystal Growth \& Design 6 (2006) 1826.

[30] M. Koini, M. Koini, T. Haber, O. Werzer, S. Berkebile, G. Koller, M. Oehzelt, M. Ramsey, R. Resel, Thin Solid Films, in print.

[31] A. C. Hillier, M. D. Ward, Phys. Rev. B 54 (1996) 14037.

[32] T. Haber, M. Oehzelt, A. Andreev, A. Thierry, H. Sitter, D.-M. Smilgies, B. Schaffer, W. Grogger, R. Resel, J. Nanosci. \& Nanotechnol. 6 (2006) 698.

[33] A. Sassella, D. Besana, A. Borghesi, M. Campione, S. Tavazzi, B. Lotz, and A. Thierry, Synth. Metals 138 (2003) 125. 


\section{Figure caption:}

Fig.1: Atomic force microscopy of a $10 \mathrm{~nm}$ thick sexiphenyl film on $\mathrm{KAP}(010)$ : elongated islands are observed with two distinct preferred growth directions inclined by an angle of $70^{\circ}$ to each other. Some areas appear dark and are not covered by needles.

Fig.2: X-ray diffraction of sexiphenyl crystals grown on $\mathrm{KAP}(010)$ : the specular scan shows the 20-3 diffraction peak of sexiphenyl (a) and rocking curves across the 030 specular diffraction peak of the substrate and of the 20-3 orientation of the organic layer (b).

Fig.3: A single pole figure taken at $2 \Theta=42.40^{\circ}$ of sexiphenyl crystals grown on $\mathrm{KAP}(010)$, concentric circles gives the $\psi$-angle in steps of 15 degrees. The three strong pole densities located at $(\psi / \varphi=) 19^{\circ} / 90^{\circ}, 19^{\circ} / 270^{\circ}$ and $75^{\circ} / 0^{\circ}$ arises from the $-140,140$ and $01-2$ reflections of the substrate. The pole densities of the sexiphenyl crystals are marked with arrows and indexed by $\{21-3\}$.

Fig. 4: Scheme of the alignment of sexiphenyl crystals on $\mathrm{KAP}(010)$ at the molecular level. The top view is in the center, while side views along the two corrugation [001] and $[ \pm 101]$ directions of the substrate are given on top and on the bottom, respectively. The orientation of the sexiphenyl molecules relative to the adapted surface corrugation is shown at the bottom. The individual molecules are drawn by van der Waals models; carbon and hydrogen atoms are drawn by light-grey and white colour, respectively.

Fig. 5: Summary of empirical force field calculations showing the potential energy for all possible in-plane alignments of sexiphenyl crystals (left) and for corresponding misorientations of the sexiphenyl (20-3) plane with the (010) surface of KAP (right). Energy has been normalized to a single p6P molecule contacting the KAP substrate.

Fig. 6: Defocussed transmission electron microscopy image of a sexiphenyl thin film grown on $\mathrm{KAP}(010)$ surface (a). The selected area diffraction pattern proves that, despite the main orientation, there are some crystallites of standing molecules (b). High resolution transmission electron microscopy images prove the single crystal nature of the elongated islands (c). 
Figure 1

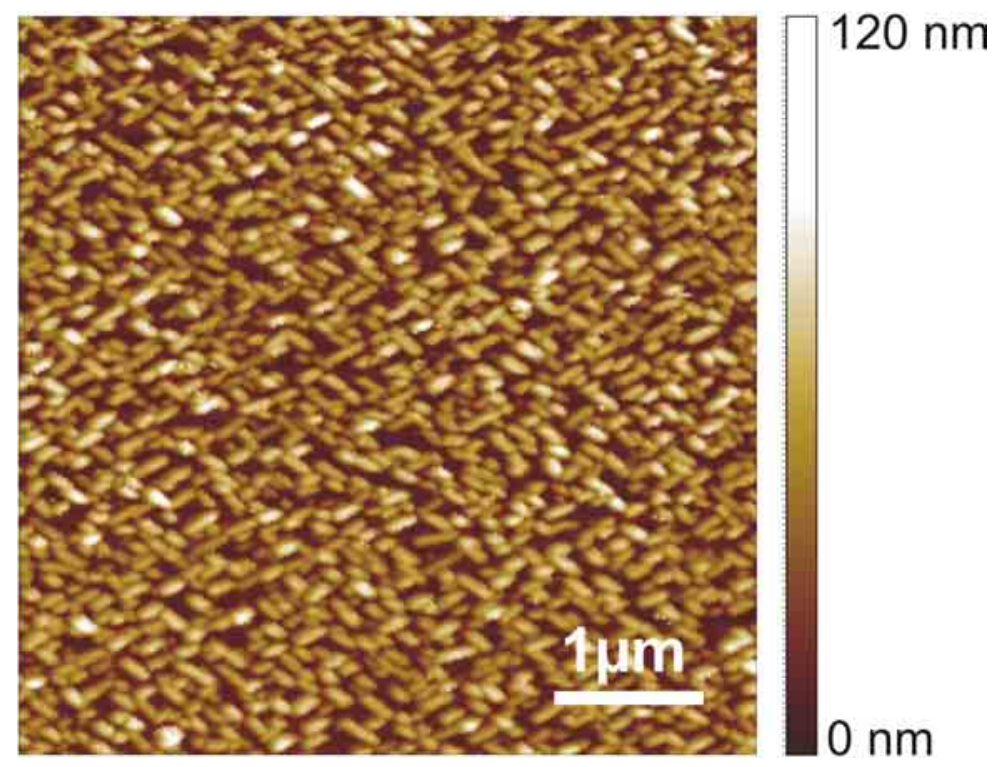

Figure 2
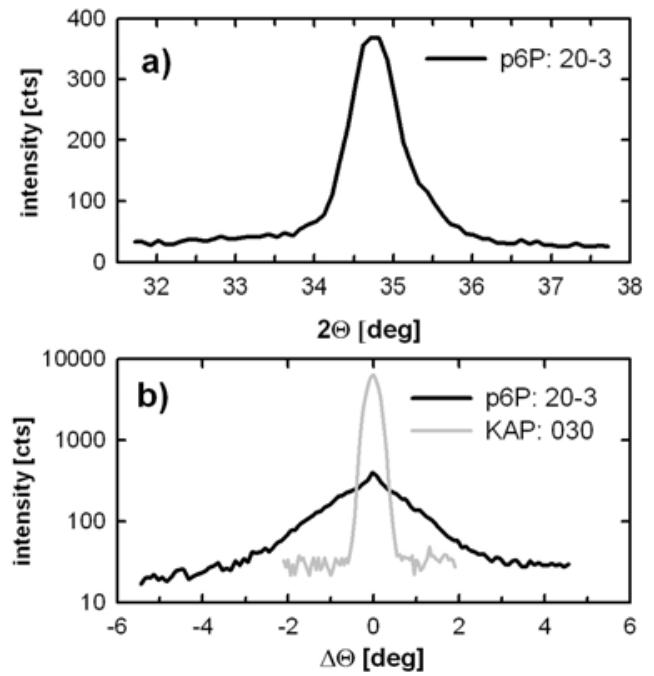
Figure 3

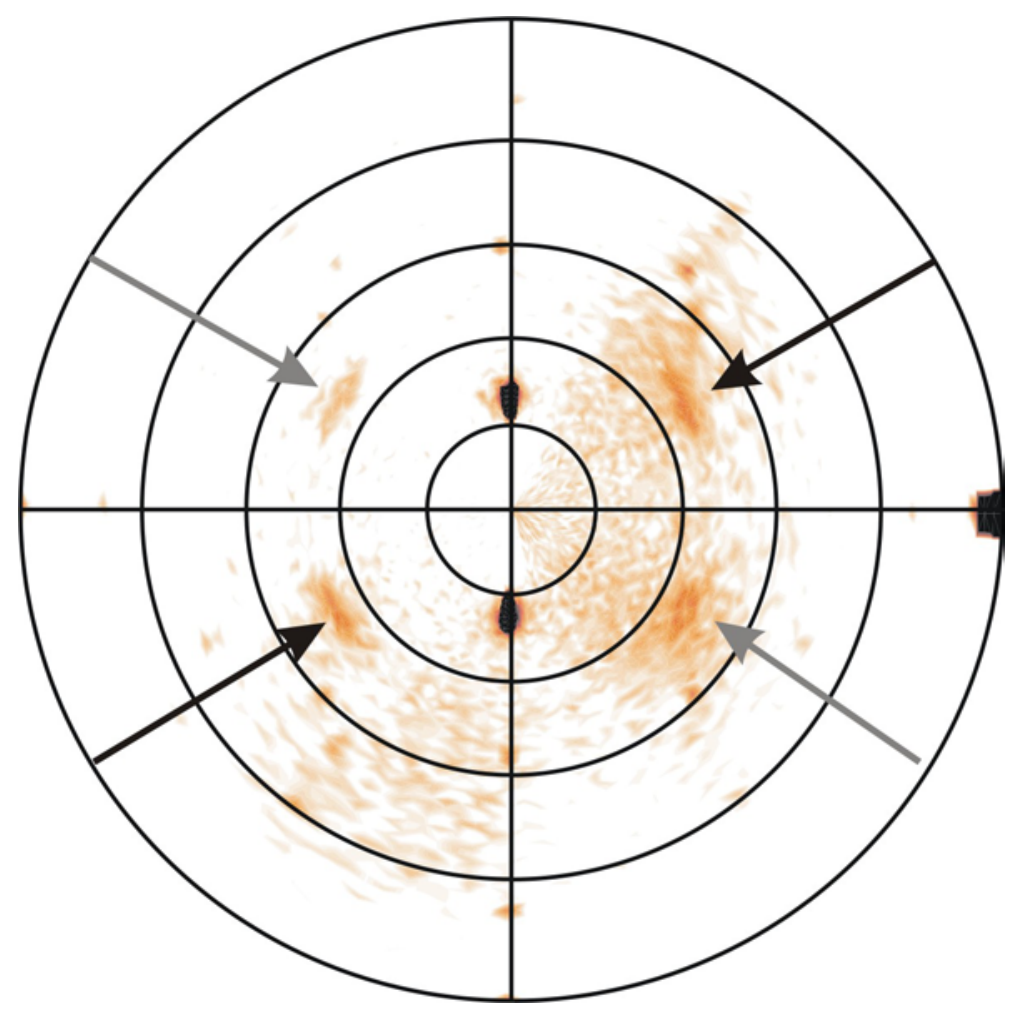

Figure 4

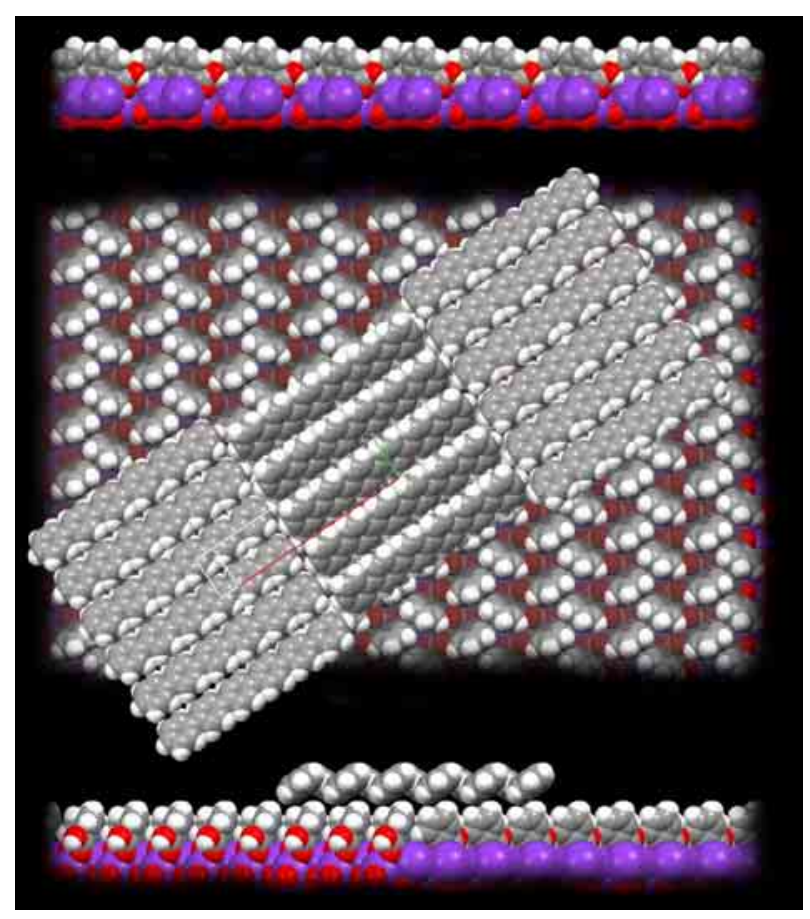


Figure 5
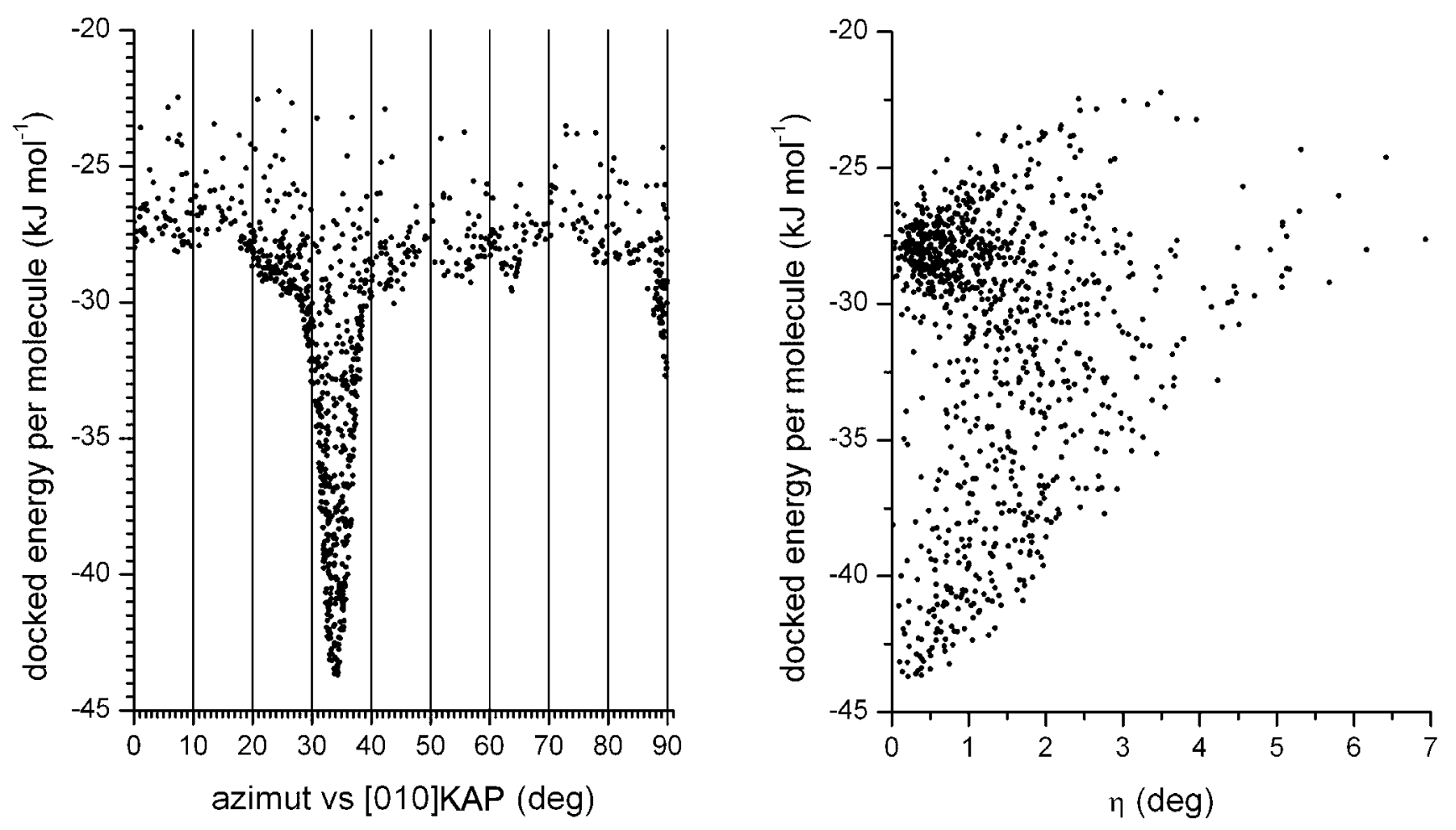

Figure 6
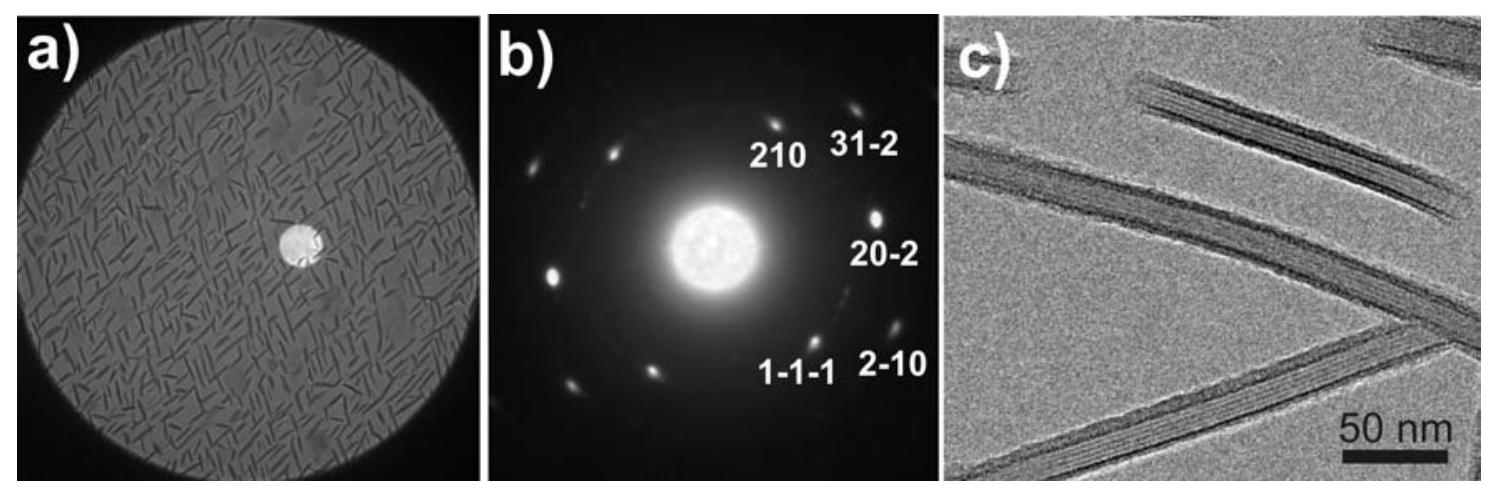